\title{
Ciencias SOGIALES Y SOCIOGRAFía ESTATAL. TRAS EL ESTUDIO DE LA FAMILIA OBRERA PORTEÑA, 1899-1932
}

\author{
Hernán GonZÁlez Bollo*
}

Estudiar los miembros que constituyeron la familia popular urbana de la ciudad de Buenos Aires en las décadas del diez y del veinte, sus ingresos y consumos, remite a toda una perspectiva historiográfica de estudios sociales, la que enfatiza la constitución y las prácticas culturales y políticas de los sectores populares. ${ }^{(1)}$ Asimismo, otra proposición historiográfica - apoyada en los presupuestos teóricos de una ortodoxia "clasista" - recuerda la necesidad de contemplar una visión del mundo de la clase obrera desde sus bases sociales. ${ }^{(2)}$ A estas orientaciones de la historia social habría que agregar la importancia histórica de la operación de conocimiento social que llevó a cabo la estadística pública argentina. En vísperas del Centenario, la compilación de indicadores sociales tales como los salarios percibidos por los obreros, las condiciones habitacionales y los consumos de la familia popular portena, constituyeron buena parte de los objetivos de los estadísticos de una flamante agencia creada por el Estado nacional, el Departamento Nacional del Trabajo (DNT).

Estos trabajos de cuantificación fueron posibles gracias al empleo de técnicas y metodologías avaladas por un uso ya extendido en las oficinas dedicadas a la misma problemática entre los países europeos, desde fines de siglo pasado. En nuestro país, como del otro lado del Atlántico, el estudio de los cambios sociales que se sucedieron en el mundo del trabajo hizo cada vez más necesaria la elaboración de modelos de asentamiento de datos mediante planillas de registro. De esta manera, los encuestadores laborales pusieron en marcha esquemas implícitos de clasifica- 
ción y jerarquización de datos sociales, realizando una operación de representación de la sociedad y de los grupos que la constituían. ${ }^{(3)}$ El estudio de las condiciones de vida de la clase obrera argentina por la sociografia estatal es un ejemplo de lo que acabamos de afirmar, pues la División de Estadística del DNT sentó las bases para su análisis cuantitativo haciendo de la unidad doméstica porteña el objeto central de esos estudios, hasta aproximadamente la llegada de la Gran Depresión.

La puesta en marcha de estas investigaciones sociales tuvo como antecedente académico la preocupación de algunos docentes de la Universidad de Buenos Aires (UBA) en el desarrollo de objetos de estudio social. Juan Agustín García fue el primero en proponer un método empírico para aprehender los matices ofrecidos por la realidad social argentina. Dos de los profesores titulares de la primera cátedra de Sociologia, creada en la Facultad de Filosofia y Letras (LBA), Ernesto Quesada y Ricardo Levene, expresaron también con variada intensidad la necesidad de experimentar las herramientas provistas por la disciplina en cuestión.

La preocupación de los docentes universitarios y la recolección de datos sobre los presupuestos obreros realizada por las primeras encuestas del DNT fueron los antecedentes de los primeros números indicadores (index numbers) del costo de la vida en el país. Este cálculo anual fue producto de la activa participación de los docentes y alumnos de uno de los seminarios de otra institución académica, la Facultad de Ciencias Económicas (UBA). Su divulgación fue posible gracias a la edición de la Revista de Economía Argentina (REA), como así también a los artículos referidos al tema publicados en la prensa escrita porteña.

Estos primeros análisis empíricos de la clase obrera porteña, mediados por la organización familiar, se corresponden con un contexto histórico singular. Los espacios institucionales y las corrientes intelectuales que hicieron posible trazar "en el papel" un nuevo grupo social emergieron en momentos de la incipiente sustitución de manufacturas importadas que sostenía el proceso industrial, cuyos efectos fueron la mayor ocupación obrera y la mutación de la escena urbana. Estas mismas condiciones sociales permitieron elaborar el primer costo de la vida, que simboliza como tal la inquietud de la opinión pública por la incertidumbre económico-social posterior a la primera guerra mundial y, a la vez, la renovación de las ciencias sociales.

\section{Sociologia y realidad social: antecedentes académicos} de la investigación social

A fines del siglo pasado, algunas corrientes intelectuales contribuyeron a una revolución en las metodologías de las ciencias sociales en las que una aproximación inductivista paulatinamente cobró reconocimiento académico frente al enfoque deductivista que presuponía la existencia de leyes y principios generales inherentes a la conducta social. ${ }^{(4)}$ Juan Agustín García, Ernesto Quesada y Ricardo Levene se 
preocuparon por concretar un conocimiento social empírico, en el que cada uno de ellos privilegió una metodología diferente de investigación reconocida en el Viejo Mundo. ${ }^{(5)}$

En polémica con las aproximaciones metafisicas, García se propuso retomar la tradición de estudios sociales de Esteban Echeverría, Vicente Fidel López, Bartolomé Mitre y Juan Bautista Alberdi, para introducirla en la UBA y demostrar a sus estudiantes que era posible formar una ciencia social argentina. Si se quería una sociología nacional el primer elemento de investigación era la familia argentina, "el alma del complicado tejido social", según García. ${ }^{(6)}$ Para la descripción de ese objeto importó la metodología ideada por el ingeniero francés Fréderic Le Play (1806-82), porque consideraba que la combinación del método de la escuela histórica, con el de observación y el de muestreo estadístico servía a los fines que se había propuesto. ${ }^{(7)}$ Convencido de las virtudes empíricas de este primer modelo de encuesta social reprodujo la planilla de registro, y afirmó:

"Así, con este método, estudiamos en un contacto íntimo con la realidad. El filósofo va cargado de números y apuntes. No debe ser hombre sedentario porque su ciencia no está en los libros, sino en la calle, en los conventillos y en los ranchos". ${ }^{(8)}$

García iba más lejos, pues sostenía que la teoría jurídica implícita en el Código Civil argentino había dejado "fuera del mapa" a las clases populares; sospechaba que una observación metódica de la realidad social de nuestro país revelaría la existencia de dos o tres tipos de familias diferentes que reclamaba a los codificadores argentinos leyes originales adaptadas a sus formas de vida.

El primer plan de estudios para el doctorado de la Facultad de Filosofia y Letras aprobado el 15 de mayo de 1896 incluía en el cuarto año la asignatura "Ciencia Social"; en diciembre de 1898, reformado el plan, se cambió la primera denominación por "Sociología". El primer curso fue dictado por Antonio Dellepiane en 1898 y no volvió a dictarse hasta que en abril de 1904 se votó la terna para la provisión del cargo de profesor titular: la integraron Ernesto Quesada, José María Ramos Mejía y Juan Agustín García, en ese orden; se designó como profesores suplentes a Alfredo Colmo y Carlos Saavedra Lamas. En 8 de julio, el Consejo Directivo debió considerar una solicitud de los alumnos para que se dictara la materia y se resolvió entonces, habiéndose ya formado la terna, iniciar su enseñanza.

Ernesto Quesada inauguró el primer curso anual en 1905 denominado "La historia crítica de la sociología y su metodología". Propuso un estudio genético de la ciencia de la sociedad, un análisis de los métodos sociológicos y, posteriormente, la aplicación de algunos de ellos a los problemas sociales del Nuevo Mundo, a partir del modelo de seminario. ${ }^{(9)}$ Dos años más tarde, Quesada dictó una conferencia pública en la ciudad de La Plata en la que trató de demostrar las virtudes de las herramientas sociológicas al proponer el estudio empírico del "problema nacional obrero" en la Argentina. No polemizó con las posiciones metafísicas sino con el sentido común criollo y el "incómodo desgano" de algunos miembros del parla- 
mento y del gobierno. ${ }^{(10)}$ Estaba convencido de que el vacío temático no lo cubrieron ni la investigación de Juan Bialet Massé, ni el "meritorio" trabajo de Juan A. Alsina, ni la obra de José Ingenieros. ${ }^{(11)}$ En cambio, propuso a su auditorio mostrar cómo funcionaba un laboratorio de ciencias sociales, un seminario dedicado a la cuestión obrera apoyado en una biblioteca de obras estadísticas y monografias de Estados Unidos y países europeos.

Quesada arraigó este estudio social a explicaciones económicas. Aunque no dejó de advertir los variables alcances de las investigaciones especializadas que realizaron los estadísticos de aquellos países. Por ejemplo, el Bureau of Labor de los Estados Unidos se había ocupado de las formas que revestía el casamiento y el divorcio de la familia obrera de las ciudades industriales de la costa este; el Labour Department de Gran Bretaña habia publicado en sus Abstract of foreing labour statistics una investigación especial sobre el "sweating system" en las ciudades de Londres y Leeds; por último, la Direction du Travail francesa había estudiado la pequeña industria de la alimentación y confección, con arreglo a los métodos de la escuela de Le Play.

El caso de Ricardo Levene es notablemente diferente, ya que su tesis doctoral para acceder al grado de doctor en leyes compendió las más reconocidas teorías sociológicas de su tiempo. ${ }^{(12)}$ Tras la renuncia de Quesada en 1922 fue durante un cuarto de siglo profesor titular de la cátedra de Sociología, de la Facultad de Filosofia y Letras (UBA). A lo largo de la década del veinte, Levene consideró el problema del método de la ciencia de la sociedad y se apoyó en la escuela creada alrededor de Emile Durkheim, para dedicarse a la investigación histórica hispanoamericana. ${ }^{(13)}$ En momentos en que bajo el decanato del filósofo Coriolano Alberini se había aprobado la ordenanza de creación del Instituto de Sociología Argentina, buena parte del programa de la cátedra giró alrededor de los trabajos de Albert Bayet, Charles Blondel, Célestin Bouglé, George Davy, Paul Fauconnet y Maurice Halbwachs. Como profesor titular de la Facultad de Humanidades de la Universidad de La Plata, Levene invitó a Bouglé - entonces director adjunto de la Escuela Normal Superior de París- ${ }^{(14)}$ quien desarrolló una exposición centrada en las relaciones entre la filosofia y la sociología francesa. (15)

Al llegar al final de la década del veinte, un conjunto de docentes universitarios se planteó realizar trabajos empíricos sobre la realidad social argentina. Sin embargo, estos investigadores hoy son reconocidos sólo por sus trabajos históricos y no como precursores de una sociología empírica. A ello se debe sumar la hipótesis que sostiene que la investigación empírica no pudo concretarse por el peso excesivo del pensamiento filosófico en la ciencia de la sociedad. ${ }^{(16)}$ Es necesario considerar entonces la existencia de otros marcos institucionales - y otras condiciones materiales- para reconstruir esos primeros trabajos con base estadística. 


\section{Los estudios de campo del Departamento Nacional del Trabajo, 1913-29}

A fines de 1907, bajo la jurisdicción del Ministerio del Interior se creó el DNT, por ley $\mathrm{N}^{2} 8899$; el decreto reglamentario contempló tres divisiones administrativas, Legislación, Estadística e Inspección y Vigilancia, más los Consejos del Trabajo y el Registro de Colocaciones. ${ }^{(17)}$ También, la edición mensual del Boletín del DNT que estaba reservada para publicar folletos, monografias "o cualquier trabajo sobre asuntos que se relacionen con la cuestión social".(18)

En las décadas del diez y del veinte los fondos presupuestarios para el DNT se incrementaron apreciablemente, a la par de una ampliación de sus funciones. Sin embargo, disminuyen los recursos humanos de la División de Estadística, según se observa en el cuadro 1. Al final del período estuvo formada apenas por un jefe, un oficial, tres auxiliares compiladores y un escribiente.

Desde 1913 hasta 1930, se realizaron en total diez estudios por muestreos en barrios populares que abarcaron algo más de cuatro mil seiscientas familias. ${ }^{(19)} \mathrm{De}$ cada una de estas monografias se deducía una media llamada "presupuestos obreros-promedio".

Estas encuestas comenzaron con la llegada de Alejandro Bunge a esta División del DNT. Activo militante de los Círculos de Obreros Católicos - bajo el influjo intelectual de Emilio Lamarca- ${ }^{(20)}$ Bunge incorporó las ideas sostenidas por Le Play y sus discípulos cuando focalizó la familia popular en las monografias oficiales. En éstas criticó a los compiladores de censos nacionales y municipales, por incapacidad para establecer relaciones de parentesco:

\footnotetext{
"En los censos nacionales y municipales se han usado fichas individuales sin establecer el vínculo de parentesco de cada censado con el jefe de la familia, como se hace en todos los censos. Tampoco el tercer censo nacional, que actualmente se elabora, contendrá tales datos... No poseyendo datos censales directos, he debido calcular las cifras requeridas relativas a las familias, haciendo uso de los métodos de asociación". (21)
}

Al concluir su segundo estudio de campo en 1914, Bunge delimitó dos unidades domésticas urbanas: los hogares populares que realizan tareas en su domicilio ("sweating system") (22) y aquellos cuyos ingresos provienen del trabajo en establecimientos manufactureros. Además, seleccionó los barrios porteños de Barracas, La Boca y Liniers para las futuras encuestas al comprobar que la proporción de la población obrera era superior al resto de los barrios. Su ascenso a la jefatura de la Dirección General de Estadística de la Nación, a mediados de 1916, no impidió que continuaran los trabajos de campo de esa División: dejó organizada una metodología de trabajo con los presupuestos obreros-promedio.

En los comienzos de estos relevamientos los funcionarios del DNT no lograron discriminar los gastos populares ante la resistencia de los encuestados a dar infor- 
mación. (23) En las últimas encucstas esta reticencia disminuyó de forma tal que poco a poco pudieron conocerse más ampliamente gastos tales como vestimenta, viáticos, pago de asociaciones de mutualidad, calzado, periódicos o tabaco. Este cambio expresaría un espacio social creado entre la intención compiladora de los encuestadores laborales y la voluntad popular por dar a conocer sus ingresos y sus gastos.

\section{Morfologia de la familia porteña}

Tomando los promedios de las seis primeras encuestas realizadas entre 1913 y 1924 , los encuestadores del DNT dedujeron que la composición de la familia popular porteña era de un promedio aproximado de cinco personas. ${ }^{(24)}$ Lo confirmaron en el estudio inmediatamente posterior con un millar de familias relevadas; esta composición numérica encabezó el informe:

"Las diversas encuestas efectuadas por este Departamento han demostrado que la familia obrera está constituida, término medio, por cinco miembros: padre, madre y tres hijos; también se comprobó que de cada familia, dos son las personas que contribuyen a la formación de los ingresos, mediante su trabajo". (25)

Este último dato - dos miembros trabajan- era la garantía del éxito de las unidades domésticas en cubrir los gastos o realizar pequeños ahorros:

"Se observa que en los 38 casos en que las entradas superan a los gastos, el $40 \%$ de los individuos que constituyen esas familias son los que trabajan; en los 44 casos en que se nota déficit entre los ingresos y los egresos, sólo trabajan el 27,2 \%. En los 2 únicos casos en que el presupuesto se equilibra, trabaja el $45,4 \% "$. (26)

En la encuesta de 1924, señalaron que en la mayoría de los casos todavía dos eran las personas proveedoras de ingresos, aunque casi en la mitad había un solo "contribuyente":

"Puede decirse entonces, que en la mitad de los casos (46\%) el único contribuyente es el padre solo; por orden de mérito le sigue el padre y uno o varios hijos. Estos representan el 25.6 por ciento; figurando con el diez por ciento, los esposos. En general. dos es el número de personas que trabajan en cada familia". ${ }^{277}$

Según las encuestas posteriores eran cuatro los miembros promedio de una familia y aún dos contribuían a formar los recursos en cada una de ellas. La diferencia con otros años está en que los barrios elegidos eran diferentes, porque habían observado una movilidad territorial de las clases populares. ${ }^{(28)}$

A pesar de la nueva distribución geográfica, interesa destacar la relación entre 
los ingresos populares y $\mathrm{el}$ número de miembros de la familia. En estos años los medios académicos internacionales comenzaron a esbozar las primeras interpretaciones económicas respecto a los factores de crecimiento demográfico y, entre ellas, las teorias culturales (por oposición a las biológicas) buscaron una conexión entre la fertilidad decreciente y la prosperidad creciente. ${ }^{(29)}$ Uno de los modelos explicativos sostuvo que el mayor bienestar era el resultado del descenso de la tasa de natalidad: se limitaban los nacimientos con objeto de alcanzar la prosperidad. ${ }^{(30)} \mathrm{A}$ este clima de ideas no escaparon los demógrafos argentinos cuando trataron de explicar la menor cantidad de hijos como una alternativa popular a las mayores aspiraciones de confort. En un estudio sobre el progresivo descenso de la fertilidad conyugal en la ciudad de Buenos Aires, el subdirector de la estadística nacional, Raúl Prebisch, confirmó la disminución del número de hijos de las familias porteñas. El funcionario puso el acento en la mayor aspiración de las familias numerosas de rodearse de recursos, como uno de los obstáculos para lograr una mayor natalidad. ${ }^{(31)}$ Se hace más evidente esta relación - la adquisición de bienes y el control de la natalidad- ante un mercado renovado por la mayor oferta de artículos, producto de las inversiones de origen norteamericano, entre 1921 y 1930. ${ }^{(32)}$

\section{Alimentos y otros gastos}

Las primeras encuestas reconocieron tres precios diferentes para los mismos alimentos: los de los "abastecedores" (que hoy día llamamos mayoristas), los de venta al menudeo (más conocidos como minoristas) y "los precios reales que paga el obrero". (33) Con esta última forma de compra popular los encuestadores configuraron un tipo de obrero sin capacidad de cálculo económico. En este relevo se cumple la "ley" que había sido propuesta por el estadistico prusiano Ernst Engel (1821-96), según la cual la parte de los consumos alimenticios de los presupuestos aumenta cuando los ingresos disminuyen. Según el sofisticado registro de la alimentación realizado en 1925 , poco más de la mitad del presupuesto obrero estaba destinado a este rubro. ${ }^{(34)}$

Los dos estudios realizados en 1913 y 1922 mostraron que el 20 y $25 \%$ de los ingresos se invertían en "otros gastos". (35) En esta última encuesta se discriminaron los gastos de luz. Este dato debe asociarse a la lenta extensión de la red eléctrica, a partir de la década del veinte en los barrios populares. ${ }^{(36)}$ La llegada de electricidad acompañó otro proceso ligado a la urbanización: la expansión de la red de tranvías con la consiguiente baja de la tarifa. ${ }^{(37)}$ La electrificación del transporte y su extensión intercomunicó los barrios que, junto al consumo eléctrico domiciliario, posibilitó las condiciones para la universalización de las prácticas de una middle class y concretó una cultura material standard. 


\section{Dos vias hacia una vivienda confortable}

Cada estudio de campo demostró que el confort habitacional sufrió un complejo proceso y que existieron dos vías para concretar un mejor espacio doméstico. Los encuestadores tomaron como punto de partida del estudio de campo los conventillos de los barrios céntricos.

En 1919, de las 80 viviendas encuestadas más de la mitad carecían en absoluto de ventilación. ${ }^{(38)}$ En la década siguiente, diferenciaron a las viviendas populares alquiladas entre las de material y las de madera. ${ }^{(39)}$ La Memoria de la División de Estadística sostuvo que el precio del alquiler de la pieza de material en un conventillo bajó con relación a lo que se había pagado el año anterior y que las "casas chicas", es decir, de tres o cuatro piezas se mantuvieron sin variación. ¿Cuál era la razón de esta baja en las piezas históricamente de mayor demanda popular?:

"Las ventas de terrenos por mensualidades, con facilidad inmediata de edificación, ha influido poderosamente en la despoblación de los conventillos, pues con el pago del mismo alquiler, van amortizando la deuda del terreno adquirido, ya que las habitaciones, en su mayoría, las construyen de maderas". ${ }^{(40)}$

Más tarde se realizó otra encuesta de 5.000 piezas de material alquiladas que permitió establecer que poco menos de la décima parte estaban vacías. Los estadísticos del DNT advirtieron que tomando el quinquenio 1923-27 había una baja del $13 \%$ en los precios, por mes y según el número de habitaciones. Lo correlacionaron con la existencia de una mayor cantidad de permisos municipales para edificar. ${ }^{(41)}$

Se pueden deducir dos vías de concreción del mayor confort de la casa popular: la primera, era la compra de la casilla de madera cuya ventaja residía en su facilidad para desarmarla y transportarla a los terrenitos de los barrios periféricos, aunque allí no tenían luz eléctrica; la segunda, ante el alza del alquiler de una habitación aumentaron las posibilidades de mudarse a una casa de varios ambientes, aprovechando que el precio de las "casas chicas" no varió. La diferencia entre ambas vías reside en que una ofrecía a la minoría aspirar a la propiedad; y que la otra ofrecía a la mayoría alquilar una vivienda de más metros cuadrados.

\section{Los alcances metodológicos de los presupuestos-tipo del DNT}

El estudio de los presupuestos de las familias obreras con arreglo a los métodos elaborados por la escuela formada alrededor de Fréderic Le Play remite, antes de Juan Agustín García, al corazón de la militancia católica argentina de fines de siglo pasado.

José Manuel Estrada toma como modelo a Le Play, porque este católico 
conservador francés cruzaba la tradición religiosa con el racionalismo cartesiano. ${ }^{(42)}$ Bajo el control político del incipiente "tercer espacio ideológico" —antisocialista y antiliberal- que representaron los católicos sociales, el DNT recogió la información estadística del nivel de vida popular porteño. ${ }^{(43)}$ De la mano de otro católico como Alejandro Bunge se iniciaron los estudios sociográficos en nuestro pais, cuyo objetivo era arribar a un promedio de la suma de todos los casos estudiados. Con la llegada del joven universitario Bunge desde la Alemania del II Reich se importó toda una tradición de la estadística administrativa prusiana, la Statistiches Reichs Amt, afin a los métodos propuestos por Ernst Engel. ${ }^{(44)}$ Sin embargo, a mediados de la década del veinte este modelo de estudio ya no seguía las normas del Bureau International du Travaily de la Sociedad de las Naciones. Alejandro Unsain, delegado argentino a la Tercera Conferencia de Estadistica del Trabajo, defendió allí sin éxito este método. Los delegados del resto de las naciones avalaban la novedosa elaboración de presupuestos familiares, discriminando ingresos, industrias y regiones, mediante libretas repartidas entre las clases populares, desarrollado por Maurice Halbwachs. (45)

Albert Thomas - director de la Oficina Internacional del Trabajo- en una nota al ministro del Interior informó de las resoluciones de la conferencia de estadigrafos referidas a los nuevos métodos de encuesta laboral. Aunque, más bien le preocupaba conocer la existencia de alguna medida por parte del gobierno argentino para concretar un programa de normalización de los números índices del costo de la vida compatibles con el de otras naciones miembros. ${ }^{(46)}$

\section{Nace el costo de la vida}

En 1912, el DNT editó un número del Boletin dedicado a investigar el problema de la carestía de la vida de las clases populares, reproduciendo sólo tablas comparadas de precios. ${ }^{(47)}$ Seis años más tarde, se publicó la primera monografía que calculó las oscilaciones anuales del costo de la vida, desde 1910 hasta 1917.(48) El costo de la vida era una sencilla fórmula con usos sociales variables: una regla para entrenar en matemáticas aplicadas a los estudiantes avanzados para acceder al doctorado en ciencias económicas de la UBA; una fórmula que cobró importancia política al incorporar nuevas explicaciones sobre las características del alza de precios, desde fines de la década del diez; y, por último, una metodología de relativa importancia para las políticas públicas de la década del veinte, a pesar de los intentos por perfeccionarla de estadísticos estatales, provinciales y municipales.

Esta primera monografia practicó dos "rupturas metodológicas" en la historia de la medición social argentina. Primero, se introdujeron los números indicadores simples y ponderados para calcular la proporción de alimentos, otros gastos y alquiler de la vivienda popular, elaborados a partir de la consulta de la obra escrita de dos economistas del ámbito académico anglosajón y dos de origen alemán. ${ }^{(49)}$ 
Todos los que habian realizado estos cálculos se preocuparon por establecer relaciones entre el valor de compra de la moneda y los precios de los bienes, mediante el estudio de series estadísticas para conocer la magnitud del ingreso nacional. ${ }^{(50)}$ La segunda innovación inscripta en la publicación del primer costo de la vida, esbozó una definición de "raza" más inclusiva de los miembros que constituían la sociedad argentina que la propuesta por José Ingenieros. ${ }^{(51)}$ Se alejaba de la concepción de los estadios de evolución largamente popularizada por el aparato positivista:

"Entendemos por cultura, en este caso, no un determinado grado de progreso y
civilización, sino el conjunto de condiciones propias que caracterizan a una raza o a
una agrupación humana más o menos homogénea. Asi consideramos que existe una
cultura etíopc, japonesa, francesa, alemana. La nuestra - quizá podemos aspirar ya a
llamarle cultura argentina - tendrá en ínfimo grado algo de las culturas indigenas, del
territorio que hoy ocupamos, más o menos indefinidas, bastante de la cultura española
y colonial y algo de las demás de Europa, pero será distinta de todas ellas en los mismos
detalles asimilados, ....". 52$)$

\section{El seminario "Costo de la vida y poder adquisitivo de la moneda"}

Desde la creación de la Facultad de Ciencias Económicas (UBA), en 1913, se implementaron los institutos de investigación económico-social y el régimen de seminario para adaptar la experiencia de la universidad alemana y estadounidense. Una de las finalidades académicas era formar un cuerpo de docentes estable capaz de promover estudios prácticos, combatir el enciclopedismo, aminorar la importancia de las conferencias y obtener el mayor fruto del trabajo personal del docente y sus alumnos. ${ }^{(53)}$

El decano de esta facultad, Eleodoro Lobos, al inaugurar los cursos del año 1919 enfatizó que la segunda monografia publicada sobre el costo de la vida era resultado de estas orientaciones. ${ }^{(54)}$ Lobos se refirió al seminario "Costo de la vida y poder adquisitivo de la moneda", dirigido por Raúl Prebisch, en el que los alumnos recogieron estadísticas en estudios de campo, realizaron una nueva investigación de precios en la ciudad de Buenos Aires, compararon sus oscilaciones en distintas provincias del país, recopilaron números indicadores formulados en distintos países y en diversas épocas, y distinguieron los salarios reales de los nominales de la ciudad de Rosario, de 1900 a 1919. (55) Como consecuencia de estas investigaciones los tres rubros que conformaban la fórmula del costo de la vida cambiaron. ${ }^{(56)}$

El campo académico argentino desarrolló una herramienta de interpretación de fenómenos económico-sociales que se popularizó durante la carestía de los años de la primera postguerra entre los legisladores nacionales y municipales, así como en la prensa escrita que los trabajadores leían. 


\section{La política y la prensa diaria tras el costo de la vida}

El primer proyecto legislativo que propuso una investigación del alza de los precios de los productos de consumo masivo era de 1912.(57) Con la publicación de la primera tabla del índice del costo de la vida aumentaron sustancialmente los proyectos de ley de los diputados nacionales, para controlar el alza. ${ }^{(58)} \mathrm{La}$ intendencia municipal porteña llegó aun más lejos que los proyectos legislativos, pues elaboró diagnósticos específicos y propuso algunas soluciones no sólo en relación a la provisión de productos de consumo masivo. Proyectó la "municipalización" parcial de la venta de artículos de primera necesidad y la promoción de las cooperativas en los barrios porteños. Ante la carestía del alquiler de la vivienda, realizó un estudio en el que comparó los metros cuadrados edificados y la cantidad de permisos municipales en la década del diez y reconoció la existencia de un problema de oferta ante la falta de construcción de edificios al finalizar los festejos del Centenario. ${ }^{(59)}$

Una de las combinaciones más significativas en estos años entre la política y la prensa escrita era la de los socialistas porteños con la edición de La Vanguardia. Allí se elaboró una defensa del consumidor, argumentando que el alza del costo de la vida se originó en el proteccionismo arancelario a la producción regional y en la existencia de trusts que especulaban con la escasez de productos de gran demanda. Por ejemplo, se denunció el privilegio que poseía la producción azucarera, como parte de las maniobras políticas que favorecian a los monopolios de los "señores del azúcar", defensores de un proyecto oligárquico en el país que explotaba a los consumidores. ${ }^{(60)}$ Con el alza del precio del pan los columnistas socialistas analizaron los componentes de la cadena productiva y de ella dedujeron una triple explotación: los acaparadores de trigo, los molineros y los dueños de las panaderías. ${ }^{(61)}$ Más aún, denunciaron la capacidad de "grandes trusts" de sustraer de circulación grandes cantidades de productos para producir un alza mecánica de los mismos. ${ }^{(62)}$ En cambio, el medio socialista aceptó el alza del precio de la carne en el consumo interno dada la influencia de la mayor demanda del comercio exterior. ${ }^{(63)}$

Las denuncias socialistas dieron paso a las propuestas, tanto de los legisladores como de los concejales socialistas. En el ámbito partidario se organizó el "Comité pro defensa de los consumidores", mediante comisiones vecinales en barrios porteños. En el recinto parlamentario, el diputado Nicolás Repetto reivindicó la alternativa cooperativa con apoyo municipal. En la Comisión Especial Pro Abaratamiento de la Vida del Concejo Deliberante, el concejal Spinetto propuso seis preguntas que organizaron la investigación. Las fuentes de información eran los artículos sobre el costo de la vida, los Boletines del comercio exterior de la Dirección General de Estadísticas de la Nación y del DNT. (64)

La Prensa era el diario de mayor lectura popular por sus avisos clasificados. En un editorial de mediados de 1917, este matutino repitió algunos de los argumentos 
socialistas sobre las causas del alza de los precios. ${ }^{(65)}$ Entre 1919 y 1921 , momento de los crecientes precios de los alimentos, vestimenta y vivienda, publicó varios artículos sin firma sobre algunas consecuencias del mayor costo de la vida. (66)

Entonces se necesitaron $\$ 168$ para adquirir los mismos productos que antes de la guerra se habían obtenido con $\$ 100$; la carestía generalizada había quebrado - aun en las clase acomodadas- la sensación de vivir en la abundancia, se cambiaron los hábitos aunque se conservaron bienes de consumo cotidiano, no se desperdiciaba nada en la producción agropecuaria y se clasificaron los residuos industriales. ${ }^{(67)}$ Emilio Lahitte, estadigrafo de la Dirección de Economía Rural y Estadística del Ministerio de Agricultura, observó una baja en el consumo de productos con base en harinas blancas del $15 \%$ entre 1919 y 1920, respecto al promedio de 1916 y 1918; la suba de los precios del pan y los fideos provocó la sustitución de su consumo por frutas y legumbres. ${ }^{(68)}$ La compilación de series anuales de la producción de bienes alimenticios permitió observar que cl pan lideró el consumo popular, le seguía la carne, la leche, el vino, el azúcar, la cerveza, la yerba, el arroz, el café, el queso y la manteca.

En el otoño de 1921 se anunció en el país un hecho novedoso y singular: la lenta deflación de los precios, cuyas causas combinaban el descenso de la capacidad productiva y el aumento de la circulación monetaria. ${ }^{(69)}$

\section{Los dilemas presentes en la Primera Conferencia Nacional de Estadistica}

Uno de los hitos más significativos en la historia de la estadística social en la Argentina fue la realización de la Primera Conferencia Nacional de Estadística, realizada en la ciudad de Córdoba a mediados de la década del veinte. El propósito de este encuentro era uniformar en todas las oficinas públicas del pais los procedimientos empleados para compilar, recoger y tabular las estadísticas existentes, sugerir los medios para perfeccionarlas y establecer los métodos más adecuados para organizar las mediciones de las que se carece parcial o totalmente. ${ }^{(70)} \mathrm{A}$ pesar de los objetivos de centralizar las mediciones que tocaban al mundo del trabajo, los asuntos referidos al costo de la vida fueron objeto de análisis en dos mesas diferentes, la Sección II, Economía y la Sección IV, Trabajo.

En la mesa de Economía se propuso a las direcciones provinciales de estadística recoger mensualmente los precios de los bienes en los principales centros urbanos bajo su jurisdicción, contemplándose las modalidades locales de consumo. Se recomendó levantar los números índices de los alimentos, vivienda y otros gastos con la fórmula ponderada, a la que ya hemos hecho referencia anteriormente. Otra regla era que, a partir de 1926, se tomaran los precios de los bienes de consumo entre el 10 y el 15 de cada mes, lapso en que habitualmente se pagaban los sueldos y quincenas de menores montos. Por su parte, la mesa de Trabajo recomendó a las oficinas provinciales similares al Departamento Nacional del Trabajo que llevaran 
una estadística relativa de los recursos y los gastos de la familia obrera, independientemente del cálculo general del costo de la vida. Asimismo, que se ampliara el estudio de bienes de consumo de uso común de la población obrera a 27 artículos para la investigación mensual de sus precios. ${ }^{(71)}$

Puede verse también en el medio académico la misma falta de unidad de criterios respecto a qué organismo estatal debía estudiar en forma conjunta las condiciones del mundo obrero y el cálculo del costo de la vida popular. Algunos de los expositores del Congreso Internacional de Economía Social, realizado por el Museo Social Argentino, propusieron estudiar las cuestiones obreras en una mesa presidida por los funcionarios del DNT; con la presencia del jefe de la Dirección General de Estadística de la Nación y de sus pares de México y Brasil, en otra mesa se discutieron los métodos y objetivos de las estadísticas sociales. ${ }^{(72)} \mathrm{El}$ espacio de discusión dispuesto por este Congreso y la Primera Conferencia Nacional de Estadística permite sopesar que el conocimiento de las necesidades populares y los métodos para su estudio social se institucionalizaron de forma separada. Más aún, a pedido de la Comisión de Legislación del Trabajo de la Cámara de Diputados, la División de Estadística del DNT ofreció las cifras anuales de la ocupación obrera desde 1914 hasta 1927, advirtiendo las dificultades para establecer un índice de los desocupados por la falta de fuentes. ${ }^{(73)}$

\section{La construcción estatal de la clase obrera argentina}

Con el fin de la próspera década del veinte concluyó la bifurcación del conocimiento institucional del mundo del trabajo. En 1929 la curva ascendente de la ocupación obrera se quebró y los encuestadores de la agencia laboral comentaron la situación de precariedad del empleo popular, al afirmar "que el remanente del personal obrero ocupado trabaja con intermitencia y no en formá continua, haciéndolo alternativamente por turnos en las industrias no paralizadas, o bien soportan interrupciones periódicas totales del trabajo en otras industrias".(74)

Tras el golpe de Estado de septiembre de 1930, se realizó otro estudio de campo que abarcó un total de 900 familias, integradas por 3.676 personas, que ocupaban 960 habitaciones. Otro signo de la grave situación popular era que por primera vez desde el comienzo de estos estudios sociales el término medio de las entradas anuales de cada familia obrera arrojó un déficit frente a los gastos promedio de sus presupuestos. ${ }^{(75)}$ Un decreto del general Agustín P. Justo encomendó definitivamente a la División de Estadística del DNT las investigaciones necesarias para calcular los índices del costo de la vida obrera y establecer las fluctuaciones de los salarios y los consumos populares. ${ }^{(76)}$ El nuevo jefe de esta División, José de Figuerola, editó el primer censo nacional de desocupados a la vez que elaboró una investigación de 5.000 familias obreras estratificadas en diez grados-tipos de salarios diferentes, según las resoluciones de la Conferencia Internacional de Estadísticas 
del Trabajo. Ello ocurría en momentos que se reseña la obra de Maurice Halbwachs, L'Evolution des besoins dans les classes ouvrières (Librairie Félix Alcan, París, 1933) en la Revista de Economia Argentina ${ }^{(77)}$ y que adquiere una gran importancia la escuela durkheimiana en los programas de la cátedra de Sociología de Levene.

Con los tiempos impuestos por la Gran Depresión, la adopción por el DNT del método desarrollado por Halbwachs cerró en la sociografía estatal el capítulo metodológico con arreglo a la obra de Le Play. Avalado por la Organización Internacional del Trabajo, el estudio de varios tipos de presupuestos obreros junto a la elaboración mensual del costo de la vida, va a ofrecer un conocimiento más uniforme de las estrategias de la familia obrera porteña, a la vez que va a brindar más matices hacia el interior del grupo social. En una década que desde el vamos se anunció sombría, los futuros estudios sociográficos formarán parte de la flamante maquinaria burocrática creada por el Estado regulador conservador para realizar un acto de nominación de la clase popular urbana con el diseño de taxonomías estadísticas surgidas de los muestreos porteños.

Los espacios académicos y estatales que se han relevado a lo largo de este trabajo, permiten reconstruir las corrientes intelectuales que nutrieron el estudio social de la clase obrera argentina en el primer tercio del presente siglo. En fin, permiten también iluminar el exclusivo esfuerzo del DNT, durante la crisis a lo largo de una década más, por realizar una operación de representación con arreglo a las técnicas estadísticas, que funcionará como soporte de las políticas públicas conservadoras y que se nutrirá del conocimiento ordinario del medio social. ${ }^{(78)}$

\section{Notas}

(1) Véase, Leandro Gutiêrrez, "Condiciones de la vida material de los sectores populares en Buenos Aires: 1880-1914", en: Revisti de Indias, № 163-164, Sevilla, 1981, págs. 167-202, que polemiza con la suposición de un crecimiento de los "salarios reales" como variable interpretativa de la prosperidad popular, sostenida por Roberto Cortés Conde en El progreso argentino, Sudamericana, Buenos Aires, 1979. También, los trabajos contenidos en Diego Armus (comp.), Mundo urbano y cultura popular. Estudios de Historia Social Angentina, Buenos Aires, Sudamericana, 1990, y de Leandro H. Gutierrez y Luis Alberto Romero, Sectores populares, cultura y política. Buenos Aires en la entreguerra, Buenos Aires, Sudamericana, 1995.

(2) Véase, Ernesto Salas y Pablo Pozzi, "Por una historia de la clase obrera", en: Contra la corriente. Historia, Teoria y Política, año 1, № 1, Buenos Aires, agosto, 1990, págs. 43-45.

(3) Cfr. Claude Grignon y Jean-Claude Passeron, Lo cullo y lo poprular. Miserabritismo y poprulismo en sociologia y en literatura, Madrid, Ediciones De la Piqueta, 1992, päg. 61.

(4) Cfr. Eduardo Zimmermann, Los reformistus liberates. La cuestión social en la Argentina, 1890-1916, Buenos Aires, Sudamericana, 1995, pág. 217.

(5) Cfr. Diego Pereyra, La enseñanza de sociología en la Universidad de Buenos Aires (1898-1921), Tesis de Maestria, Facultad de Ciencias Sociales, UBA, Buenos Aires, 1998, mimeo.

(6) Cfr. Juan Agustín García, Introlucrión al estudio de las ciencias sociales argentinas, Buenos Aires, Ángel Estrada y Cía., 1907 (4² edición), pág. 62. 
(7) García cita la obra del divulgador de Le Play, J. B. Maurice Vignes, La science sociale d'après les principes de Le Play, 2 vol., París, V. Giard \& E. Brière, 1897.

(8) Cfr. Obras completas de Juan Agustín García, Buenos Aires, Ed. Antonio Zamora, 1955, pág. 727.

(9) Cfr. Facultad de Filosofia y Letras, Progrumas de 1905, Buenos Aires, Imprenta Didot de Félix Lajouane, 1905 , págs. 5-7.

(10) Cfr. Ernesto Quesada, "La cuestión obrera y su estudio universitario", Conferencia pública de junio de 1907 en la Biblioteca de La Plata y publicada en el Boletin del Defartamento Nacional del Trabajo [Botetín del $D N T], \mathrm{N}^{2} 1$, Buenos Aires, 30 de junio de 1907, págs. 110-152.

(11) Quesada citaba a Juan Bialet Massé, Informe solme el estado de las clases olmeras en el Interior de la Repriblica, 3 vol., Buenos Aires, Imprenta y Casa editora de Adolfo Grau, 1904; Juan A. Alsina, El obrero en la Repriblica Argentina, 2 vol., Buenos Aires, Imprenta de la calle México, 1905; y, José Ingenieros, La Législution du Travail dans la Réprublique Argéntine. Lissai critique sur le projet du Ministre Gonzálean, París, Ėdouard Córnely et Cíe., Éditeurs, 1906.

(12) Hablamos de Ricardo Levene, Leyes sociologizicas, Buenos Aires, s./ed., 1907.

(13) Cfr. Hernán González Bollo y Marta Rodríguez, "Conceptos y objetivos de la obra sociológica de Ricardo Levene, 1906-30", Noveno Congreso Nacional y Regional de Histona Argentina, Academia Nacional de la Historia, Buenos Aires, 1996, pág. 1. Por oura parte, Levene ponia el estudio de Halbwachs, La classe ouvrière et les niveaux de vie (París, Alcan, 1912), en el programa de su cátedra.

(14) Cfr. Johan Heilbron, "Les métamorphoses du durkheimisme, 1920194", en: Revue française de soriologie, XXVI, N²2, París, marzo-abril 1985, págs. 214.

(15) Cfr. Célestin Bouglé, "Sociologia y Filosofia", en: Humanidudes, L. XIX, La Plata, 1929, pâgs. 11-18.

(16) Véase, Gino Germani, "La Sociología en Argentina", en: Revisla Latinoumericana de Sociologiı, $\mathrm{N}^{2} 3$, Buenos Aires, 1968, págs. 385-419.

(17) Cfr. Néstor Tomás Auza, "La legislación laboral y la complejidad del mundo del trabajo. El Departamento Nacional del Trabajo", en: Revista de Historia del Derecho, № 17, Buenos Aires, 1989, págs. 59-104.

(18) Cfr. Boletin Ofirial de la República Argentina, año XXI, N²5706, Buenos Aires, 8 de enero de 1913, pág. 71.

(19) Cfr. "Vivienda obrera, recurso y gastos", en: Boletin del DNT, N² 30, Buenos Aires, 30 de abril de 1915 , págs. 129-137; "Recurso, gastos y vivenda de la familia obrera", en: Boletín del DNT, $\mathrm{N}^{\circ} 33$, Buenos Aires, 30 de enero de 1916, págs. 201-222; "Presupuestos obreros", en: Crónica Mensual del DNT, año III, N²32, Buenos Aires, agosto 1920, págs. 507-508; "División de Estadistica. Los presupuestos obreros y su evolución en la Capital Federal en el quinquenio 1922-1926", en: Crónicu Mensual del DNT, año X, N²109, Buenos Aires, marzo 1927, págs. 1958-1959; "División de Estadística. Recursos, gastos y vivienda de la familia obrera en la Capital federal en el año 1928", en: Crónica Mensual del DNT, año XII, № 133, Buenos Aires, mayo 1929, págs. 2695-2700; "División de Estadística. Recursos, gastos y vivienda de la familia obrera en la Capital federal, durante el año 1929", en: Crónica Mensual del DNT, año XIII, N²147, Buenos Aires, mayo 1930, págs. 3142-3146.

(20) Cfr. Néstor Tomás Auza, "La propuesta del Dr. Lamarca en el $3^{2}$ Congreso Católico, 1909-1919", en: Aciertos y fracasos sociales del catolicismo argentino, t. II, Buenos Aires, Editora Docencia, 1987, pág, 203.

(21) Cfr. Alejandro E. Bunge, "La familia obrera y su vivienda en la Capital Federal", en: Estudios, t. X, $\mathrm{N}^{2} 8$, Buenos Aires, febrero 1916, pág. 81.

(22) Cfr. Alejandro E. Bunge, "El trabajo a domicilio en la Capital Federal. Informe oficial", en: Estudios, t. VII, N² 1, Buenos Aires, julio 1914, págs. 37-49.

(23) Los funcionarios afirmaban, "pues las gentes aún se mantienen rehacias a esta clase de informaciones", cfr. "Recursos y gastos de la familia obrera; año 1925", en: Crónica Mensual del DNT, año IX, N²7, Buenos Aires, enero 1926, pág. 1735.

(24) Cfr. "Recursos, gastos y vivienda de la familia obrera", en: Boletín del DNT, año VII, N² 77, Buenos Aires, mayo 1924, págs. 1300-1304.

(25) Cfr. "Recursos y gastos de la familia obrera; año 1925", Crónira Mensual del DNT, año IX, № 98, Buenos Aires, febrero 1926, págs. 1734-1736. Las cursivas son nuestras. 
(26) Cfr. "Presupuestos obreros", en: Crónica Mensual del DNI, año III, N²32, Buenos Aires, agosto 1920, pág. 508.

(27) Cfr. "Recursos, gastos y vivienda de la familia obrera", en: Crónica Mensual del DNT, ańo VIII, N²5, Buenos Aires, enero 1925, págs. 1523-1527. Las cursivas son nuestras.

(28) "En cuanto al año 1926 se ha operado un pequeño cambio... Se debe a que la encuesta correspondiente... fue realizada en una zona central, mientras que en las anteriores lo ha sido en La Boca y Barracas", cfr. "División de Estadistica. Los presupuestos obreros y su evolución en la Capital Federal en el quinquenio 1922-26", en: Crónica Mensual del DNT, año X, № 109, Buenos Aires, marzo 1927, pág. 1959.

(29) Cfr. Sydney H. Coontz, Teonias de la población y su interpretación económica, México, FCE, 1960, págs. 78-86.

(30) Sydney Coontz cita el trabajo de Roderich Von Ungern-Sternberg, The causes of the decline in the birth rate within the European sphere of civilisation, Nueva York, Cold Spring Harbor, 1931, op. cit., pág. 70.

(31) 'El deseo de mantener y elevar el 'standard of life' parece prevalecer actualmente entre los móviles que determinan a contralorear los nacimientos". Cfr. Raúl Prebisch, "Anotaciones demográficas. A propósito de la teoria de los movimientos de la población, parte III: El equilibrio demográfico", en: Revista de Economía Argentina, t. XVIII, N²107, Buenos Aires, mayo 1927, pág. 407.

(32) En estos años entraron al país 43 grandes empresas, de las cuales el mayor número se concentraron en las ramas quimicas (13), metales (7) y artículos eléctricos (10). Se producían, entre otros bienes, discos de fonógrafo, equipos de radio, productos farmacéuticos, equipos eléctricos, jabón y artículos de tocador, productos de maíz, plomo, colas, gomas, cierres relámpago, polvo de hornear y artículos de algodón. Cfr. Javier Villanueva, "El origen de la industrialización argentina", en: Desarrollo Éconómico, vol. 12, N4 47, Buenos Aires, octubre-diciembre 1972, págs. 462-463.

(33) "Los precios de los abastecedores y mayoristas son relativamente uniformes, y tomados como indices de las oscilaciones en el costo de la vida, nos puede conducir a apreciaciones erróneas. De estos precios disfrutan algunos consumidores y son precisamente los más pudientes. Los precios corrientes minoristas son los precios reales para aquella categoria social que por su administración y sus recursos puede comprar por unidades de envase, bien pesado y bien medido,.... Algunos obreros poseen las nociones de economía doméstica suficientes para metodizar y vigilar sus compras y revisar los precios: pero para la mayoria esta facilidad no existe. Tenemos pues, con ello una tercera forma de compra en la que ya no se obtiene el carbón ni por bolsas, sino por kilos, y muchos artículos por fracciones de kilo o simplemente por unidades de dinero: "lo que den" por 10 o 20 centavos de yerba, azúcar, carne, etc.". Cfr. "Precios de los artículos de primera necesidad", en: Boletín del DNT, N²3, Buenos Aires, enero 1916, págs. 223.

(34) Las dos terceras partes se componian de carne, vino, pan, leche y huevos; el tercio restante de fideos, verduras, grasas, azúcar, papas, arroz, aceite y harina.

(35) Bajo esta denominación se compiló: vestido, combustible, vinos y licores, tabaco, libros y diarios, cuota de las sociedades obreras, diversiones y gastos por enfermedad.

(36) Cfr. Jorge F. Liernury Graciela Silvestri, "El torbellino de la electrificación. Buenos Aires, 1880-1930", en la obra de los autores, El umbral de la metrópolis. Transformaciones técnicas y cultura en la modemización de Buenos Aires (1870-1930), Buenos Aires, Sudamericana, 1993, pág. 69.

(37) A partir de 1909, se habia unificado la tarifa en 10 centavos que "representaba cerca del $4 \%$ del jornal de un peón o $2 \%$ del de un obrero especializado de la construcción". Cfr. James Scobie, Buenos Aires del centro a los barrios, 1870-1910, Buenos Aires, Solar, 1986, pág. 228.

(38) Gran parte de ellas se componían de una sencilla habitación; 19 tenían puerta y ventana, 14 puerta y banderola y 47 solamente puerta.

(39) Al ampliar la muestra a 1.000 familias en 1925 , sobre 1.057 piezas alquiladas sólo 132 eran de madera ( $\$ 22,73$ promedio) y las 925 restantes de material $(\$ 30,77$ promedio).

(40) Cfr. "Alquiler", en: Crónica Mensual del DNT, año IX, N²105, Buenos Aires, septiembre 1926, pág. 1874.

(41) Cfr. "Valor locativo de la vivienda en la Capital Federal", en: Crónica Mensual del DNT, año XI, $\mathrm{N}^{2}$ 124, Buenos Aires, junio 1924, págs. 2408-2410. 
(42) Cfr. Juan Manuel Estrada, "Le Play y el liberalismo", en la obra del autor, Discursos pronunciados por José Manual Estrada (1862-1890), Buenos Aires, Compañía Sud-Americana de Billetes de Banco, 1905, págs. 271-302.

(43) Una denuncia socialista del control católico del DNT se encuentra en Eduardo Zimmermann, Los reformistas liberales, op.cit., pág. 206. Respecto a la construcción del tercer espacio ideológico, véase Lila Caimari, Perón y la Iglesia Católica. Religion, Fstado y sociedad (1943-1955), Buenos Aires, Ariel, 1995, pág. 40.

(44) Respecto a la tradición estadistica germana, hemos consultado, Ian Hacking, "Un capitulo de estadística prusiana", en: La domesticación del azar. La erosión del delerminismo y el nacimiento de lus ciencias del caos, Barcelona, Gedisa, 1995, págs. 270-284.

(45) Cfr. "Informe del Dr. Unsain, como delegado a la Tercera Conferencia de Estadistica del Trabajo", en: Crónica Mensual del DNT, año X, № 117, Buenos Aires, noviembre 1927, págs. 2219-2228.

(46) Cfr. "Nota del director de la Oficina Internacional del Trabajo sobre la Tercera Conferencia de los Estadigrafos del Trabajo", en: Crínica Mensual delDNT, año XI, N 128, Buenos Aires, octubre 1928, págs. 2519-2520.

(47) Cfr. Boletín del DNT, N²1, Buenos Aires, noviembre 1912.

(48) Cfr. Alcjandro E. Bunge, "Costo de la vida en la Argentina, de 1910 a 1917", en: Revista de Economía Angentina [REA], L. I, N² 1, julio de 1918, Buenos Aires, págs. 44-45.

(49) Cfr. José Luis de Imaz, “Alejandro E. Bunge, economista y sociólogo (1880-1943)", en: Desarrollo Éconimico, vol. 14, N 55, Buenos Aires, octubre-diciembre 1974, págs. 548. Las obras de referencia fueron Arthur L. Auspitzy Richard Lieben, Recherches sur la théorie du prix, Paris, M. Giard \& Briere, 1914 [traducido del alemán por Louis Suret]; Arthur L. Bowley, Elements of Statistic, Londres, King \& Son, 1907; Irving Fisher, The Purshusing power of Money, its determination and relation to credil interest and crises, Nueva York, The Mac Millan Co., 1913.

(50) Véase, Eric Roll, Histonia de las doctrinas económicas, FCE, México, 1973, págs. 467-475.

(51) Desde mediados del siglo pasado y principios del presente, raza, nacionalidad y cultura eran sinónimos, véase Eduardo Zimmermann, "Raza, medicina y reforma social", en: A. Lafuente, A. Elena y M. L. Ortega (eds.), Mundialización de la ciencia y cultura nacionah Madrid, Ediciones Doce Calles, 1993, págs. 580. Un ejemplo se encuentra en la definición de la "nacionalidad argentina" de Ingenieros, que suponia sólo a las razas europeas que inmigraban y que al adaptarse al territorio nacional recibian la influencia del conjunto de variaciones sociales y psicológicas de la "Naturaleza argentina", véase José Ingenieros, "Nacionalidades y razas", en su obra Sociologia argentina, Buenos Aires, L. J. Rosso, 1918 ( $7^{2}$ edición), págs. 433-458.

(52) Cfr. Alejandro E. Bunge, "Costo de la vida en la Argentina, de 1910 a 1917", op. cit., págs. 44-45. (53) Véase, Carlos Rodriguez Etchart, "Prólogo", en: Facullad de Ciencias Económicus de la UBA. Investiguciones de seminario, vol. I, Buenos Aires, Talleres Gráficos del Ministerio de Agricultura de la Nación, 1917, págs. III-XXIV.

(54) Véase, Alejandro E. Bunge, "Costo de la vida en la Argentina, sus variaciones de 1910 a 1918", en: Anales de la Facultud de Ciencias Económicas, t. I, Buenos Aires, 1919, págs. 79-100. El decano de la Facultad de Ciencias Económicas observó que "sin desconocer la importancia de las investigación sobre salarios y costo de la vida que debemos al Departamento Nacional del Trabajo ..., he creído que debía ofrecerse a nuestros alumnos la oportunidad de colaborar en la que realiza el señor ingeniero Alejandro Bunge de manera permanente, y este profesor ha tomado a su cargo el curso correspondiente, continuando en nuestra Facultad el trabajo iniciado en la Revistu de Economia Argentini", cfr. "Discurso del Dr. Eleodoro Lobos al inaugurar los cursos de 1919", en: REA, t. I, N²10, Buenos Ares, abril 1919, págs. 269-270.

(55) Cfr. "Informe sobre el seminario Costo de la vida y poder adquisitivo de la moneda", en: Facultad de Ciencias Económicas, Investigaiones de seminario, vol. II, Talleres Gráficos Argentinos de L. J. Rosso y Cía., Buenos Aires, 1921, págs. 449-457.

(56) La proporción de rubros en 1917 era: $50 \%$ en alimentos, $20 \%$ en alquiler y $30 \%$ en otros gastos. A partir de 1919 se cambió sus proporciones a: $50 \%$ en alimentos, $26 \%$ en alquiler $y 24 \%$ en otros gastos. 
Véase, Juan Carlos Valle y Ludovico Ferrari, "Costo de la vida en la Argentina, de 1910 a 1919. Números indicadores", en: Revista de Economín Argentina, t. IV, N²2, Buenos Aires, abril 1920, págs. 253-261.

(57) Cfr. Diario de Sesiones de lu Cämara de Diputados, t. I, Buenos Aires, L. J. Rosso, 1912, pág. 477 y ss.

(58) Aunque estos proyectos tuvieron escasa posibilidad de prosperar fuera del recinto original, es interesante relevar sus alcances: la compra por parte del Estado de los artículos de subsistencia básicos, de Ricardo Caballero y José A. Montes; la propuesta de un régimen de abaratamiento de los artículos de consumo, de Francisco Beiró y Carlos J. Rodríguez; el proyecto de dotar al Poder Ejecutivo del poder para fijar los precios máximos y mínimos de dichos artículos, por Rogelio Araya, Arturo Goyeneche y Carlos Melo que, con diferencia de enunciado, lo propusieron Arturo Bas y Ricardo J. Davel. Cfr. Diario de Sesiones de la Camara de Diputados, t. II, Buenos Aires, L. J. Rosso, 1918, págs. 513 y 782. También, t. Ill, pág. 152, y t. IV del año 1920, pág. 636.

(59) Véase, Intendencia Municipal de la Capital, Aburatamiento de la alimentaciön y vivienda. Mensajes y proyectos, s./ed., Buenos Aires, 1920, y un comentario periodístico de los proyectos en "Extenso despacho de la comisión especial encargada de estudiar las medidas conducentes al abaratamiento de las subsistencias", La Prensu, 14/09/1919, pág. 11.

(60) Cfr. "El azúcar. La única solución del problema", La Vanguardia, 02/07/1919, pág. 1, y "La sanguijuela proteccionista", La Vanguardia, 13/07/1919, pág. 1.

(61) Cfr. Redacción, "El pan”. La Vanguardia, 04/08/1919, pág. 1.

(62) Cfr. "El hambre", Ia Vanguardiu, 09/08/1919, pág. 1.

(63) Cfr. "La acción municipal contra la carestia", La Vanguàdia, 06/07/1919, pág. 1.

(64) Ellas fueron: 1. ¿Existe en realidad una situación de verdadera carestia de la vida en cl país?; 2 . En caso afirmativo, ¿cuáles son sus causas?; 3. ¿En qué artículos se nota especialmente?; 4. ¿Puede combatirse dicho estado de carestía?; 5 . ¿Qué medidas cree aceptables esta comisión, y en consecuencia, aconseja que adopte el Honorable Concejo?; 6 . ¿Sobre qué artículos indica las medidas generales y particulares necesarias para poder actuar en forma inmediata y eficaz?. Cfr. "Por el abaratamiento de la vida", $L a$ Vanguartia, 14/09/1919, pág. 6. A partir de la información publicada en la Revista de Comercio y Finanzas, Spinetto observó la complejidad de la carestia al reconocer el déficit mundial de la producción del azúcar como causa del alza, y gracias a otros datos alli expuestos propuso una ley que limitara la matanza de vacas para no sacrificar el stock ganadero.

(65) Sostuvo que el Estado había tenido una importante responsabilidad en el alza de precios, ya que los impuestos públicos se habian cuadruplicado entre 1895 y 1915 , y observó que la circulación monetaria se había duplicado entre 1905 y 1917; también, el gravamen aduanero alcanzó a la mitad del valor de los artículos de consumo popular importados. El editorial aceptó como veraces las denuncias de la existencia de monopolios en el interior del país para encarecer los bienes de mayor consumo y consideró la mayor demanda exterior como causa de los crecidos valores del ganado. Cfr. Editorial, "Hechos que concurren al encarecimiento de la vida", La Prensu, 09/06/1917, pág. 5.

(66) Cfr. "La carestía de la vida. Sus causas y consecuencias", La Prensa, 27/04/1919, pág. 8; "El pan. Su importancia económica y su precio", La Prensa, 17/05/1919, pág. 6; “Abaratamiento del pan. Principales aspectos del problema", La Prensa, 26/05/1919, pág. 4; “Alza de precios. Alza general, valorización parcial y carestia", La Prensa, 09/08/1919, págs. 10-11; "El vino. Producción, consumo y costo en la República", La Prensa, 02/11/1919, pág. 6.

(67) Cfr. "El despilfarro. Indicaciones de economia doméstica", La Prensa, 09/05/1919, pág. 8.

(68) Cfr. "El consumo de pan en la República", La Prensa, 23/08/1921, pág. 10.

(69) Cfr. "Los precios de los consumos. Aumento, estabilización y descenso”, La Prensa, 24/07/1921, pág. 4.

(70) Cfr. Dirección General de Estadísticas de la Nación, Recomenduciones de lu Primera Conferencia Nucional de Estudisticu, Informe $N^{*} 16$, Buenos Aires, G. Kraft, noviembre 1925.

(71) Los 27 artículos alimenticios a relevar eran: aceite común, ajies, arroz glacé, azúcar tucumana y "de refineria", fideos blancos y "amarillos", carne vacuna, cebolla, carbón de leña, grasa, harina de trigo y de maiz, huevos, grasa, kerosene, leche, pan de primera y de segunda, papas, puerros, porotos, quesos, repollo, sal, tomate, yerba mate y zapallo. 
(72) Cfr. "Congreso Internacional de Economía Social. Antecedentes y objetivos", Boletin del Museo Social Argentino, año XIII, $\mathrm{N}^{2} 40$, Buenos Aires, octubre 1924, págs. 169-293.

(73) Cfr. Crónica Mensual del DNT, año XI, N 127, Buenos Aires, septiembre 1928, págs. 2505-2510.

(74) Cfr. "Costo de la vida en la Capital Federal", en: Revista de Economia Argentina, t. XXX, N 184, Buenos Aires, octubre 1933, pág. 314.

(75) Otra pauta de la crítica situación popular era que la proporción de alimentos había trepado al $55 \%$ de los ingresos, la vivienda al $27 \%$ y otros gastos quedaban acotados al $18 \%$, cfr. "Costo de la vida en la Capital Federal", op. cit., págs. 314-315.

(76) Cfr. "Costo de la vida. Reglas para proceder a la investigación del costo de la vida de la población obrera, encuesta básica y rectificaciones periódicas", Boletin Informativo del DNT, año XV, $\mathrm{N}^{2} 163$, Buenos Aires, agosto 1933, págs. 3548-3552.

(77) Cfr. Revisla de Economía Angentina, t. XXXI, N²183, Buenos Aires, septiembre 1933, pág. 271.

(78) Respecto a la triple perspectiva estadística, política y cognitiva en el estudio de un grupo socio-profesional, hemos consultado Alain Desrosières y Laurent Thevenot, Les catégorie socioprofessionnelles, París, éditions La Découverte, 1996 ( $4^{2}$ edición).

Cuadro 1: Recursos presupuestarios y humanos del DNT, 1912-29.

\begin{tabular}{lccc}
\hline Años & Fondos del DNT & Empleados del DNT & $\begin{array}{c}\text { Empleados de la } \\
\text { Div. Estadistica }\end{array}$ \\
\hline 1912 & $\$ 165.000$ & 37 & 10 \\
1913 & $\$ 265.000$ & 37 & 10 \\
1914 & $\$ 618.320$ & 79 & 14 \\
1915 & $\$ 231.600$ & 37 & 8 \\
1917 & $\$ 218.304$ & 36 & 8 \\
1920 & $\$ 368.320$ & 67 & 6 \\
1923 & $\$ 368.320$ & 67 & 6 \\
1925 & $\$ 368.320$ & 67 & 6 \\
1927 & $\$ 396.880$ & 67 & 6 \\
1929 & $\$ 532.120$ & 86 & 6
\end{tabular}

Fuente: Néstor Tomás Auza, "La legislación laboral y la complejidad del mundo del trabajo. EI Departamento Nacionaldel Trabajo, 1912-1925", en: Revista de Historia del Derecho, $\mathrm{N}^{2} 17$, Buenos Aires, 1989, págs. 98-99.

Cuadro 2: Encuestas del DNT sobre presupuestos obreros porteños, 1913-29.

\begin{tabular}{lcccccccccc}
\hline & 1913 & 1914 & 1919 & 1922 & 1923 & 1924 & 1925 & 1926 & 1928 & 1929 \\
\hline familias & 221 & 156 & 84 & 110 & 123 & 360 & 1000 & 700 & 1198 & 680 \\
número & 1154 & 768 & 507 & 582 & 642 & 1797 & 4273 & 2772 & 4776 & 3019 \\
promedio & 5.2 & 4.9 & 6 & 5.2 & 5.2 & 4.9 & 4.2 & 3.96 & 3.98 & 4.4 \\
ingresos \$ & 1859 & 1751 & 1712 & 2150 & 2516 & 2006 & 2033 & 1995 & 2044 & 2120 \\
alimentos \% & 44.86 & 42.20 & 59.60 & 52 & 53.7 & 58.3 & 55.82 & 48 & 51.5 & 51 \\
alquiler \% & 18.72 & 18.60 & 17.80 & 19.8 & 15.15 & 18 & 18 & 22 & 22.5 & 20 \\
ouros\% & 30.02 & 30.80 & 22.60 & 28.2 & 31.15 & 23.7 & 23.34 & 30 & 26 & 29 \\
ahorro \% & 6.30 & 8.40 & & & & & & & & \\
piezas $\mathrm{N}^{2}$ & 294 & 178 & $\mathrm{~s} / \mathrm{d}$ & $\mathrm{s} / \mathrm{d}$ & 181 & 371 & 1057 & 724 & 1296 & 799
\end{tabular}

Fuente: Boletin del DNT, 1914, 1916, 1920, 1927, 1929 y 1930. 


\section{BibLIOGRAFÍA}

Diego Armus (comp.): Mundo urbano y cullura popular. Fstudios de Historia Social Argentina, Sudamericana, Buenos Aires, 1990.

Néstor Tomás Auza: Aciertos y fracusos sociales del catolicismo argentino, 2 vol., Buenos Aires, Editora Docencia, 1987.

Néstor Tomás Auza: "La legislaciốn laboral y la complejidad del mundo del trabajo. El Departamento Nacional del Trabajo", en: Revista de Historia del Derecho, № 17, Buenos Aires, 1989, págs. 59-104.

Boletin del DNT: Buenos Aires, 1907-33.

Célestin Bouglé: "Sociología y Filosofia”, Humanidades, t. XIX, La Plata, 1929, págs. 11-18.

Alejandro E. Bunge: "El trabajo a domicilio en la Capital Federal. Informe oficial", en: Estudios, t. VII, N ${ }^{\circ}$ 1, Buenos Aires, julio 1914, págs. 37-49.

Alejandro E. Bunge: "La familia obrera y su vivienda en la Capital Federal", en: Estudios, t. X, N² 8, Buenos Aires, febrero 1916, págs. 75-82.

Alejandro E. Bunge: "Costo de la vida en la Argentina, sus variaciones de 1910 a 1918 ", en: Anales de la Faculad de Ciencias Económicas, t. I, Buenos Aires, 1919, págs. 79-100.

Sydney H. Coontz: Teorias de la población y su interpretaciön económicu, FCE, México, 1960.

Roberto Cortés Conde: El progreso argentino, Sudamericana, Buenos Aires, 1979.

Alain Desrosières y Laurent Thevenot: Les catégorie socio-professionnelles, París, éditions La Découverte, 1996 ( $4^{2}$ edición).

Dirección General de Estadisticas de la Nación: Recomendaciones de la Primera Conferencia Nacional de Estculistica, Informe N²16, G. Kraft, Buenos Aires, noviembre 1925.

Juan Manuel Estrada: Discursos pronunciados por José Manual Estrada (1862-1890), Buenos Aires, Compañía Sud-Americana de Billetes de Banco, 1905.

Facultad de Ciencias Económicas (UBA): Investiguriones de seminario, 2 vol., Buenos Aires, Talleres Gráficos del Ministerio de Agricultura de la Nación, 1917-1921.

Facultad de Filosofia y Letras: Programas, Buenos Aires, 1905-33.

Juan Agustín Garcia: Introducción al estudio de las ciencias sociales argentinas, Buenos Aires, Ángel Estrada y Cía., 1907 ( $4^{2}$ edición).

Gino Germani: "La Sociologia en Argentina", en: Revistu Latinoamenicana de Sociologria, N² 3, Buenos Aires, 1968, págs. 385-419.

Hernán González Bollo y Marta Rodríguez: "Conceptos y objetivos de la obra sociológica de Ricardo Levene, 1906-30", Noveno Congreso Nacional y Regional de Historia Argentina, Academia Nacional de la Historia, Buenos Aires, 1996.

Claude Grignon y Jean-Claude Passeron: Lo culto y lo popular. Miserabilismo y populismo en sociologia y en literatura, Madrid, Ediciones De la Piqueta, 1992.

Leandro H. Gutierrez: "Condiciones de la vida material de los sectores populares en Buenos Aires: 1880-1914", en: Revisla de Indias, N²163-164, Sevilla, 1981, págs. 167-202.

Leandro H.Gutiérrezy Luis Alberto Romero: Sectores populares, culturay politica. Buenos Aires en la entreguerru, Buenos Aires, Sudamericana, 1995.

Ian Hacking: La domesticación del azar. La erosión del detemminismo y el nacimiento de las ciencias del caos, Barcelona, Gedisa, 1995.

Maurice Halbwachs: La classe ouvrière et les niveuux de vie, París, Alcan, 1912.

Maurice Halbwachs: L'Evolution des besoins dans les classes ouvriéres, Paris, Librairie Félix Alcan, 1933.

Johan Heilbron: "Les métamorphoses du durkheimisme, 1920-1940", en: Revue francaise de sociologie, XXVI, Nº 2, París, marzo-abril 1985, págs. 203-237.

José Luis de Imaz, "Alejandro E. Bunge, economista y sociólogo (1880-1943)", en: Desarrollo Económico, vol. 14, N2 55, Buenos Aires, octubre-diciembre 1974, págs. 543-564.

José Ingenieros: Sociologia argentinłh Buenos Aires, L. J. Rosso, 1918 ( $7^{\circ}$ edición).

Ricardo Levene: Lyes sociológicas, Buenos Aires, s./ed., 1907. 
Jorge F. Liernur y Graciela Silvestri: Ll umbral de la metröpolis. Transformaciones lécnicas y cultura en la modernización de Buenos Aires (187(2-1930), Buenos Aires, Sudamericana, 1993.

Revista de Economía Argentina, Buenos Aires, 1918-33.

Eric Roll: Historia de les doctrinas económicus, México, FCE, 1973.

Ernesto Salas y Pablo Pozzi: "Por una historia de la clase obrera", en: Contra la corriente. Historia, Teoriay Politica, año 1, N 1, Buenos Aires, agosto 1990, págs, 43-45.

James Scobie: Buenos Aires del centro a los barrios, 1870-1910, Buenos Aires, Solar, 1986.

Javier Villanueva: "El origen de la industrialización argentina", en: Desurollo Económico, vol. 12, № 47, Buenos Aires, octubre-diciembre 1972, págs. 462-463.

Eduardo Zimmermann: "Raza, medicina y reforma social”, en:A. Lafuente, A. Elenay M. L. Ortega (eds.), Mundialización de la cienria y cultura nacionah, Madrid, Ediciones Doce Calles, 1993.

Eduardo Zimmermann: Los reformistas liberales. I a cuestión social en la Argentina, 18901916, Buenos Aires, Sudamericana, 1995. 